\title{
Seal Analysis for the Ares-I Upper Stage Fuel Tank Manhole Covers
}

\author{
Dawn R. Phillips \\ NASA Marshall Space Flight Center \\ Robert J. Wingate \\ NASA Marshall Space Flight Center
}




\section{Objective}

Assess the feasibility that the Naflex seal used with the Space Shuttle External Tank manhole covers can be used for the Ares-I design 


\section{Outline}

Problem description

Background

Approach for Upper Stage analyses

Analyses and results

Summary 


\section{Ares-I Upper Stage LH2 Tank}
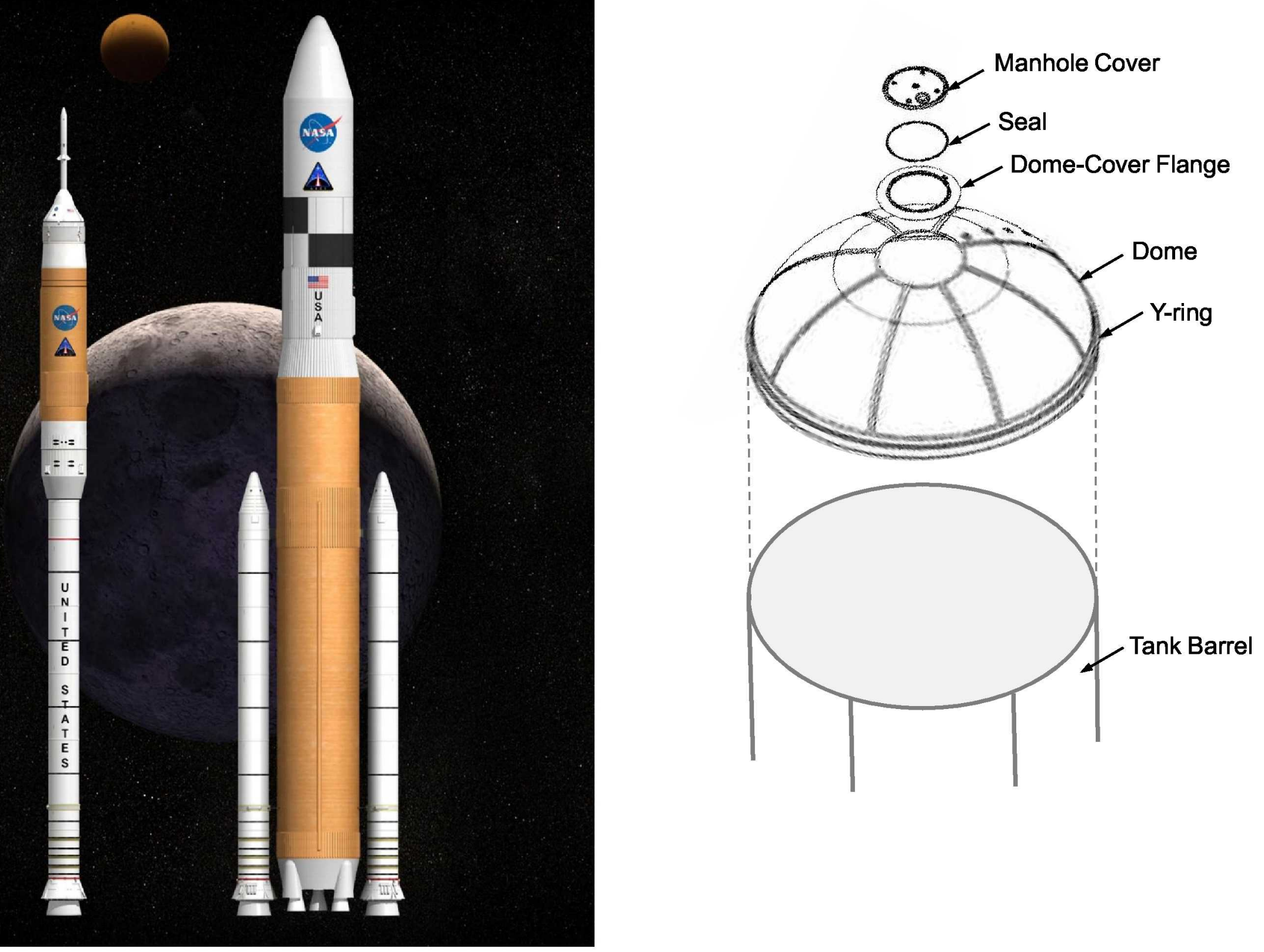


\section{Naflex Pressure-Assisted Seal}
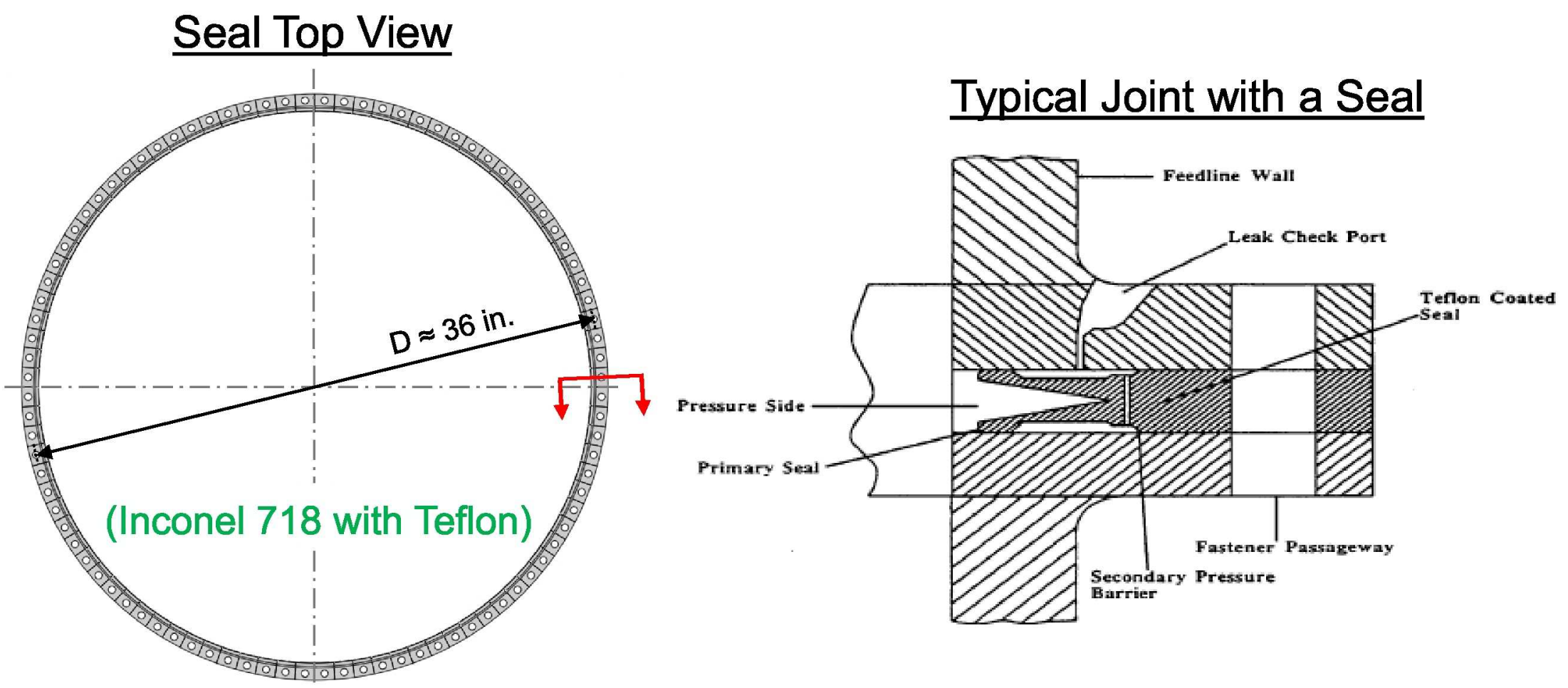

Seal Section View

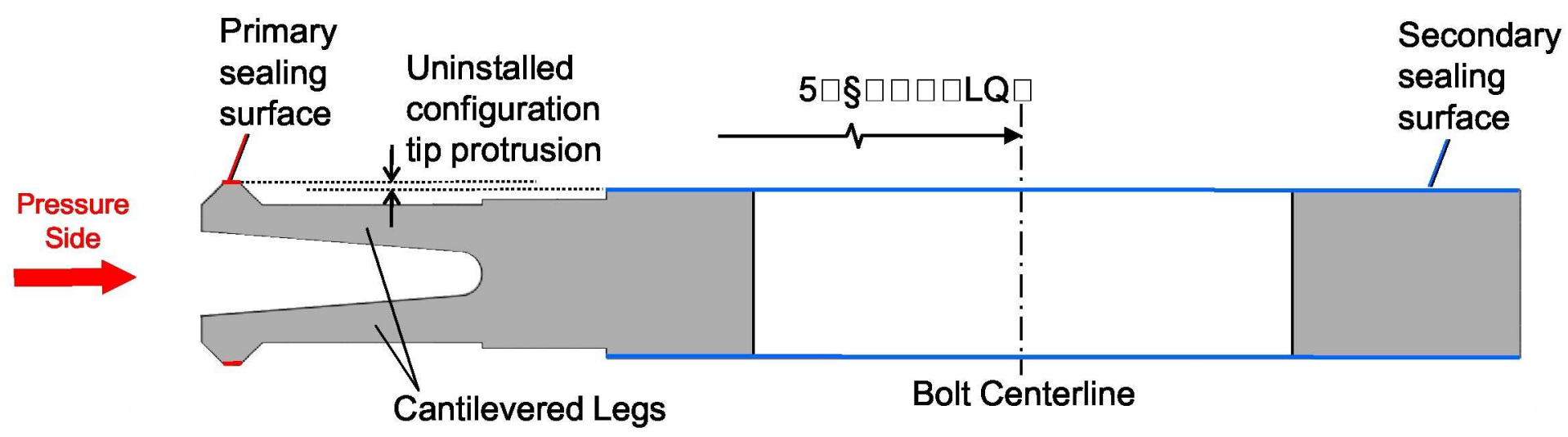




\section{Background}

- Naflex seals have long history of use in launch vehicle components, including Saturn stages and Space Shuttle External Tank

- Ares-I Upper Stage tank pressures are higher than ET pressures, requiring performance verification of heritage seal design in new manhole cover configuration 


\section{Approach for Upper Stage Analyses}

Heritage ET analyses reviewed for potential application to Upper Stage 


\section{ET Seal Test}

\section{Test Set-Up}

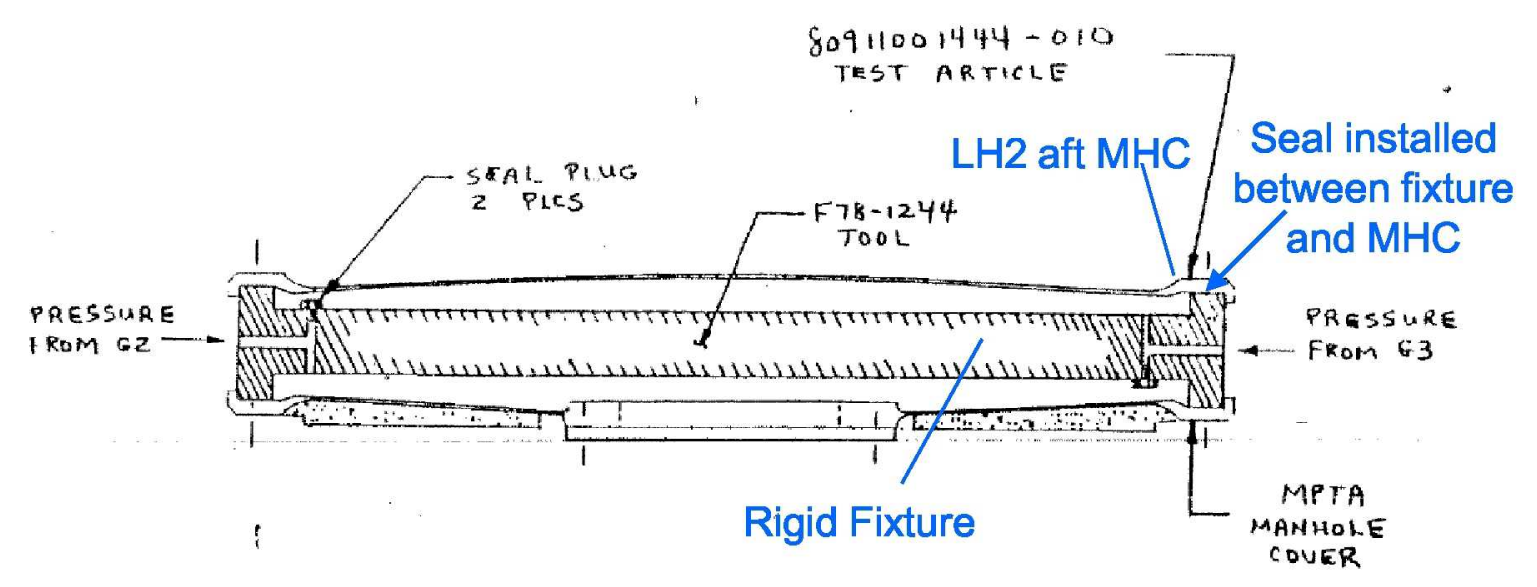

\section{Deflection Gauge Locations}
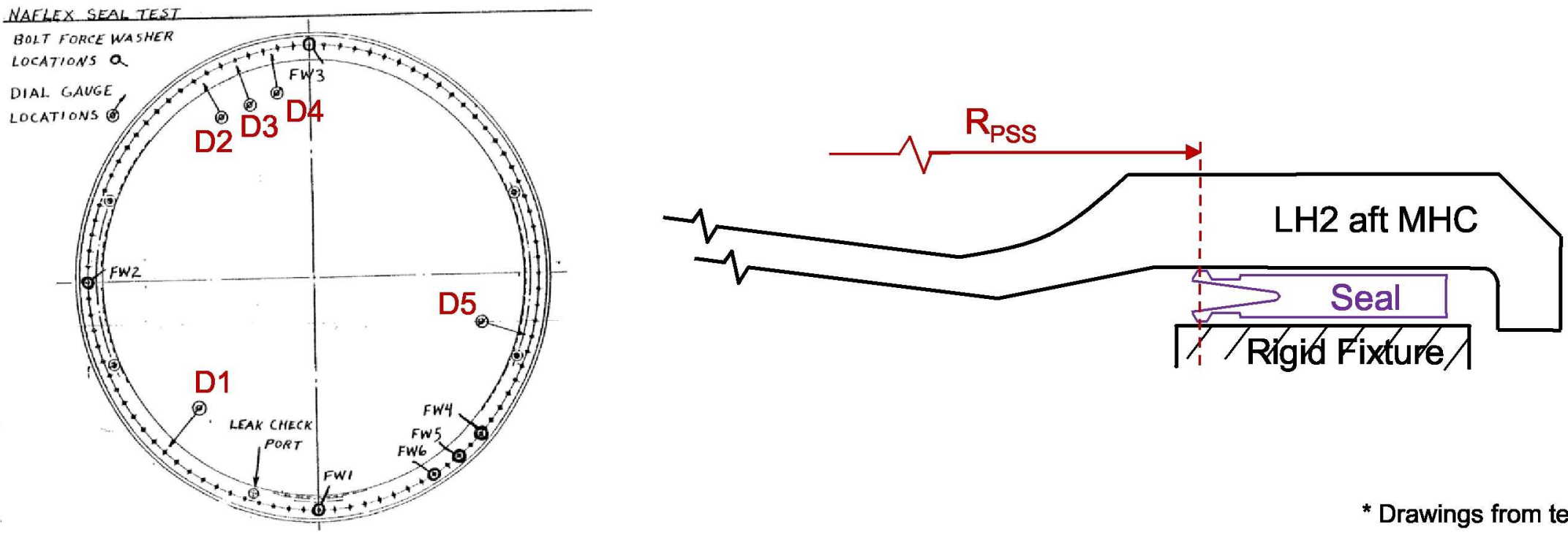

* Drawings from test report 


\section{ET Seal Test}

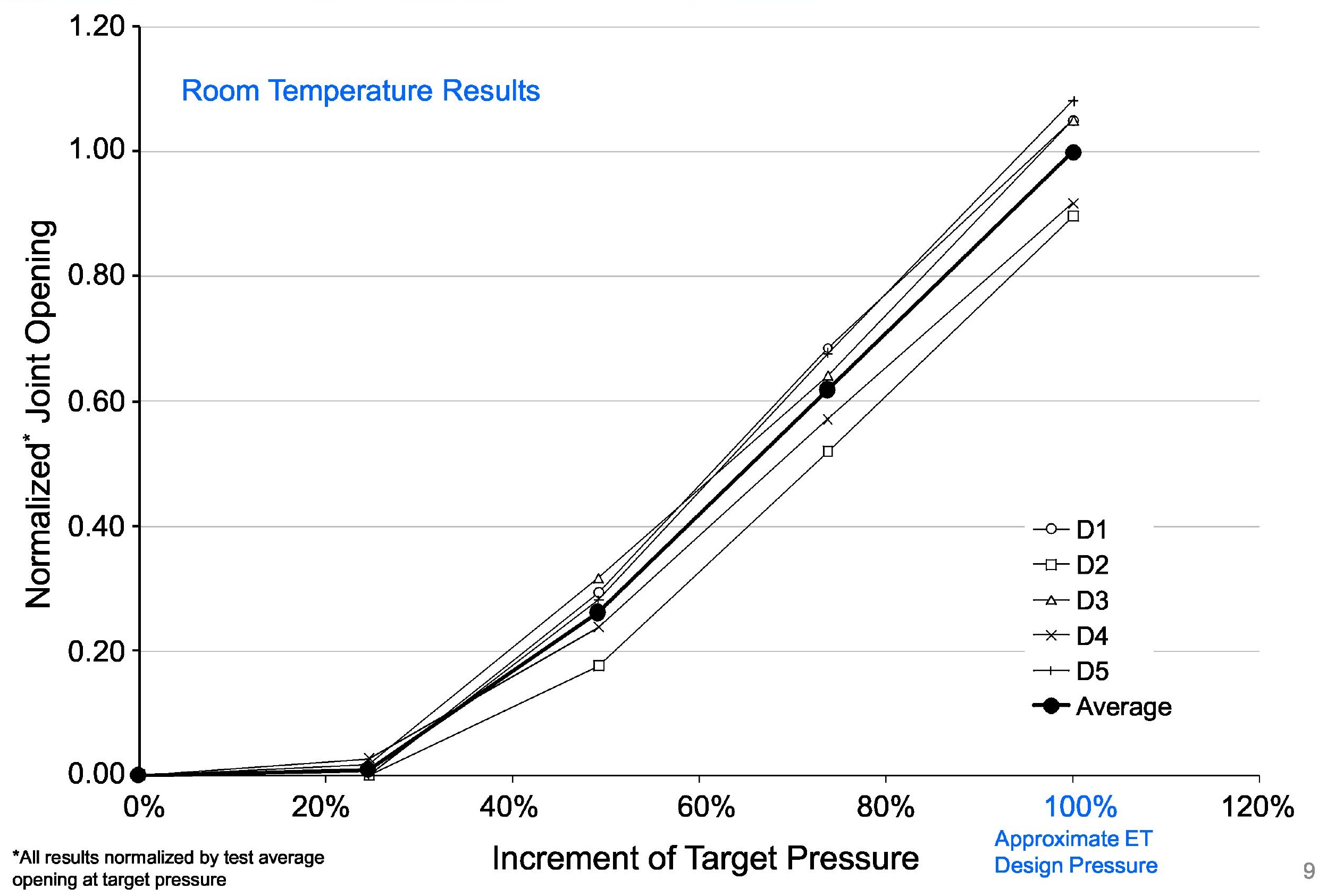




\section{D Symmetric Wedge of Naflex Seal}
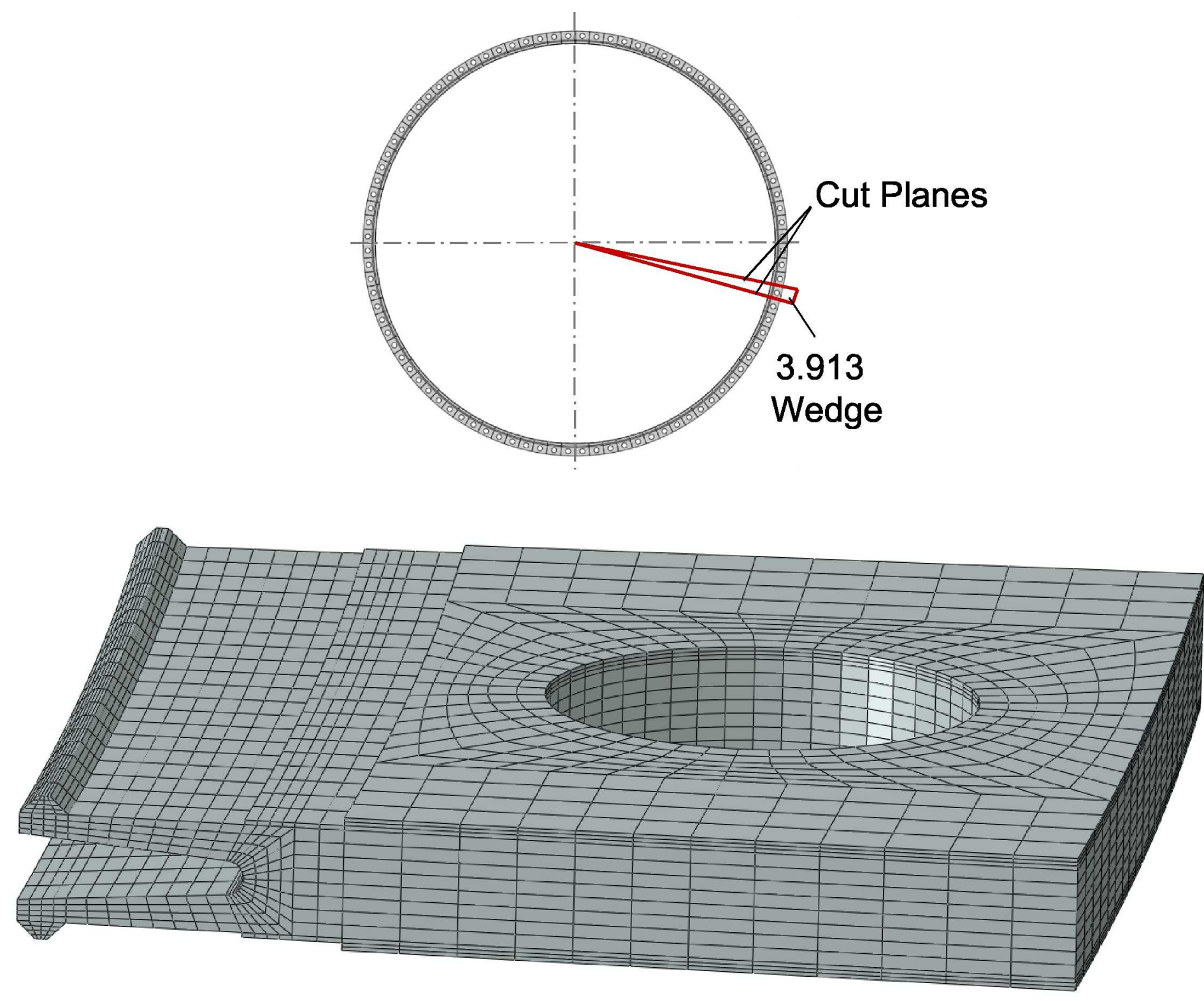


\section{ET Seal Test Finite Element Model}

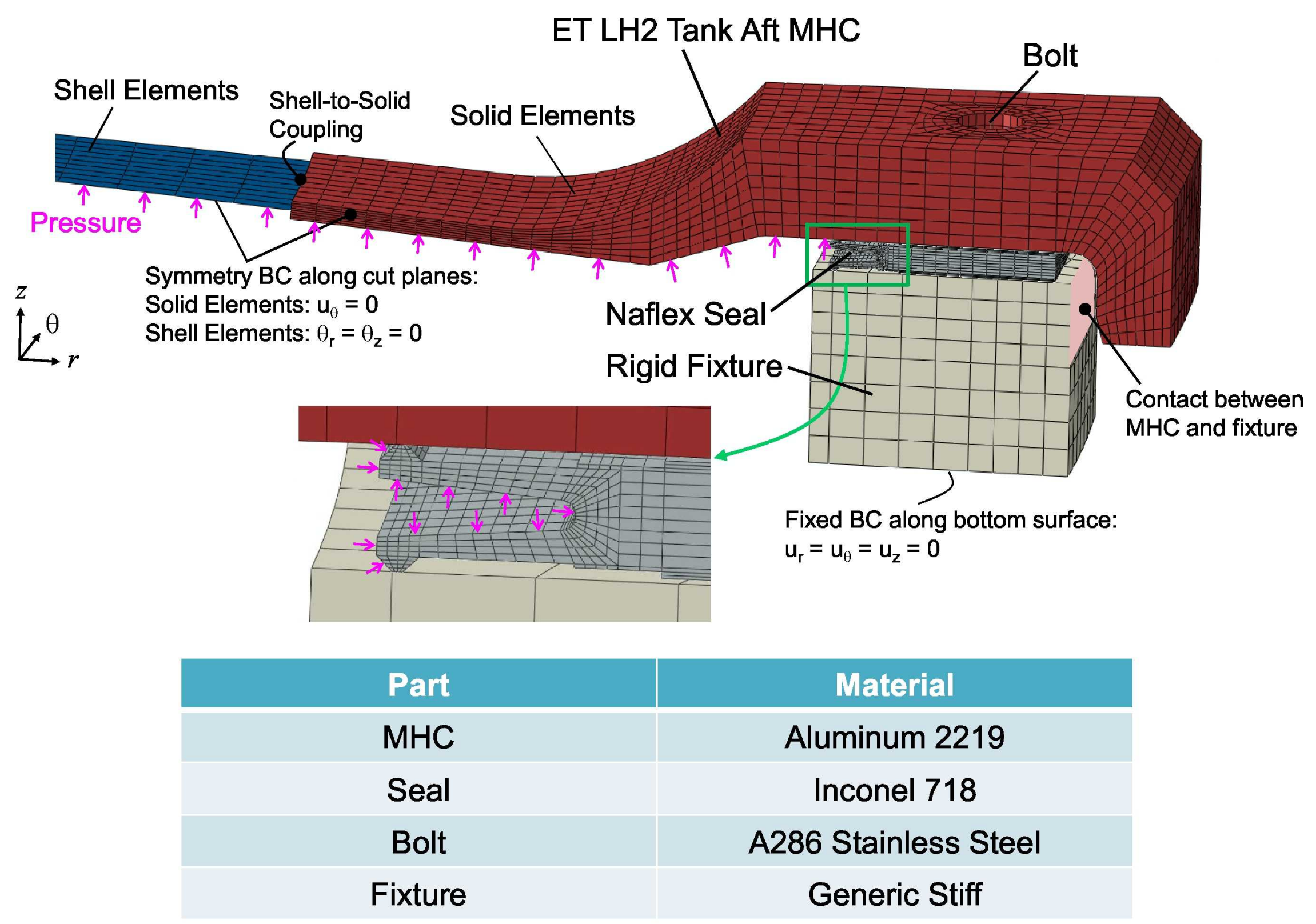




\section{ET Seal Test Finite Element Model}

\section{Bolted Joint in ET MHC}

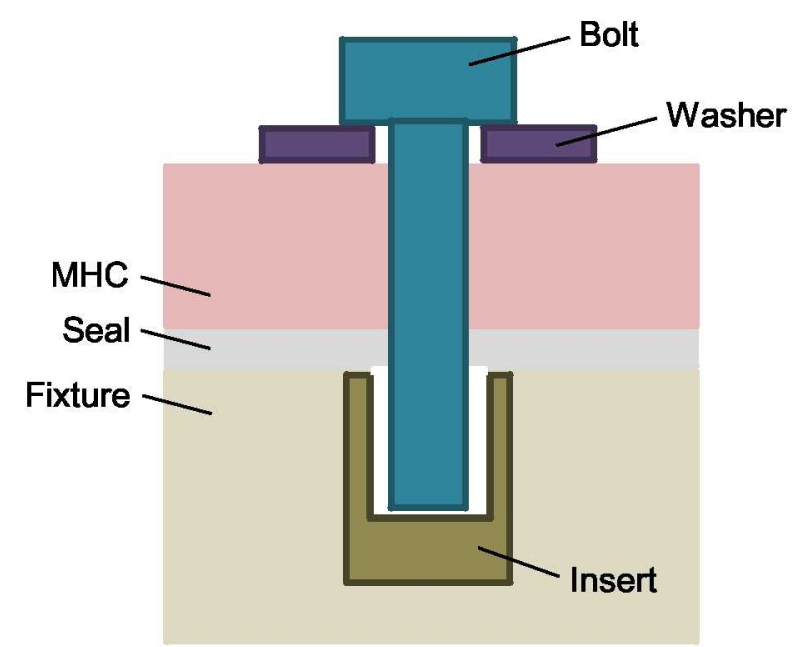

\section{SLGHU' $\square$ \&RQVWUDLQWV}

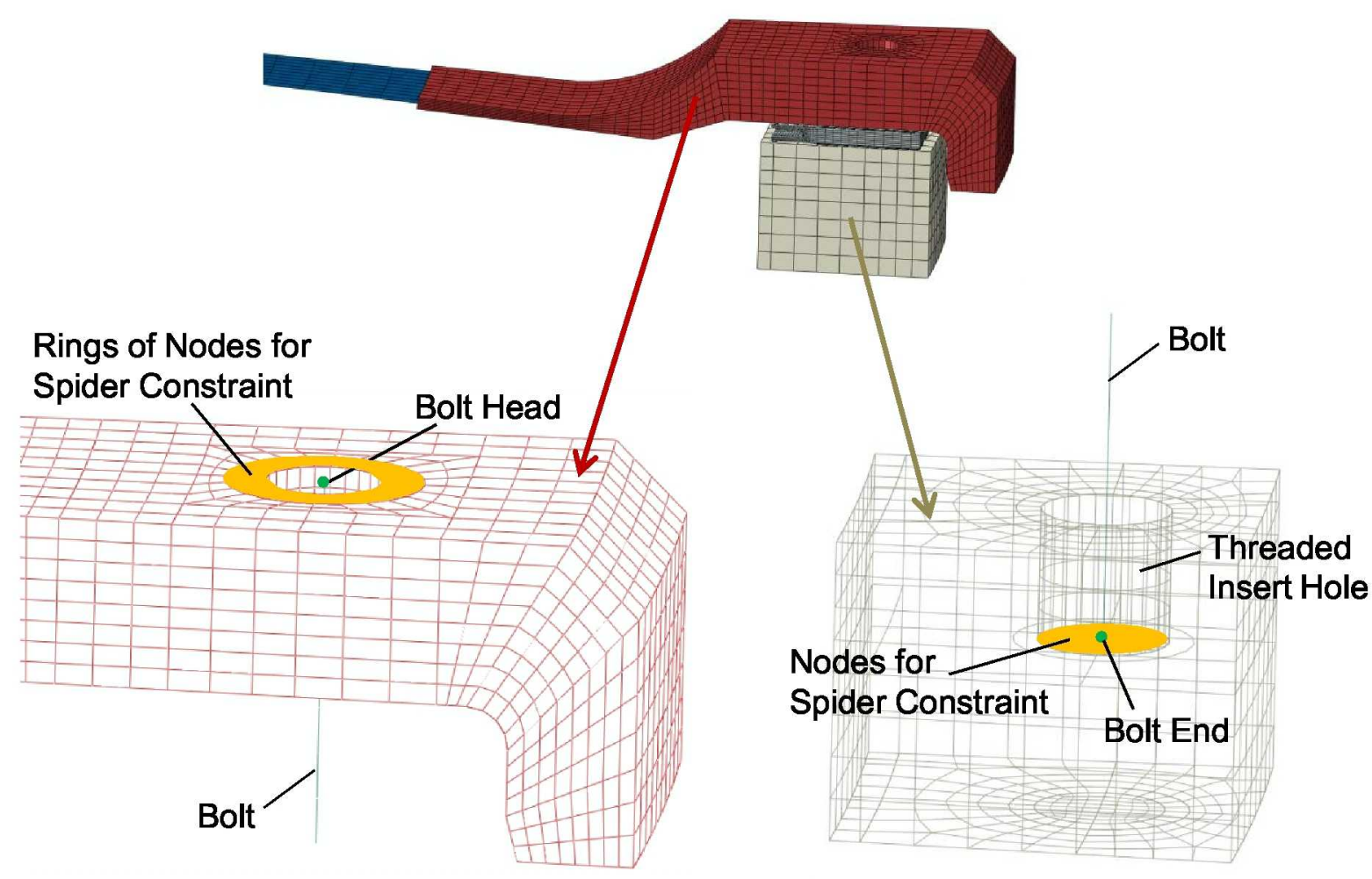




\section{ET Seal Test Finite Element Model}

$\underline{\text { Contact }}$

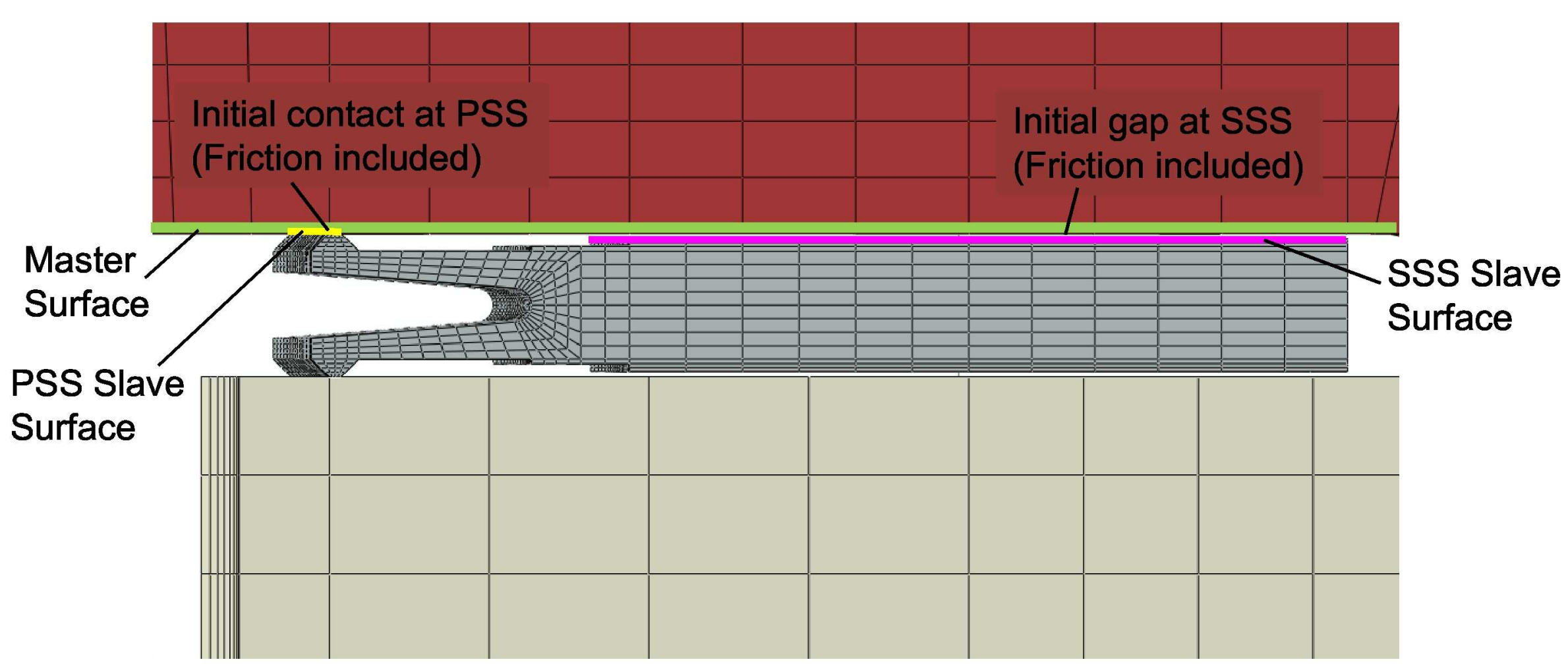




\section{ET Seal Test-Analysis Correlation}

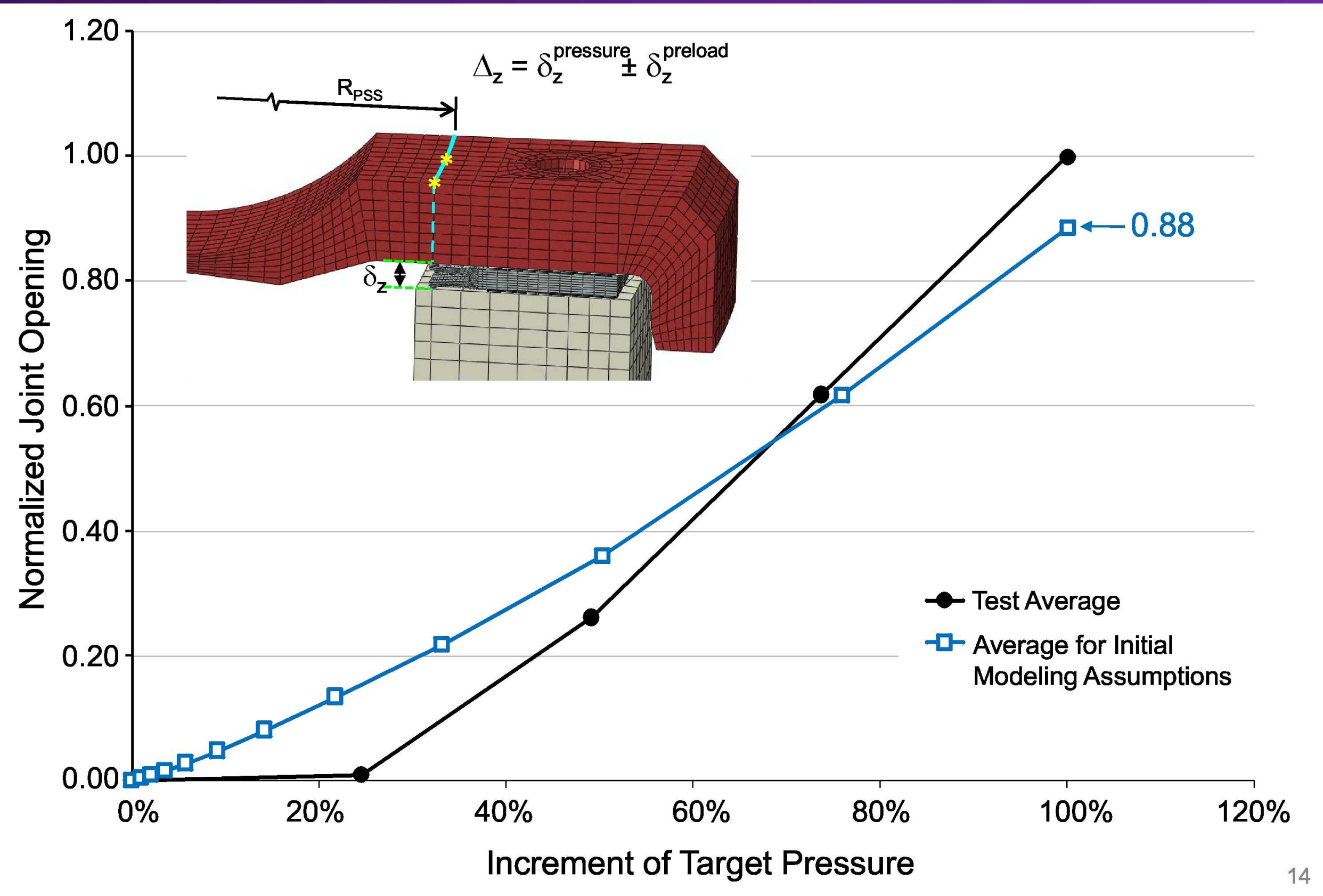




\section{ET Seal Test-Analysis Correlation}
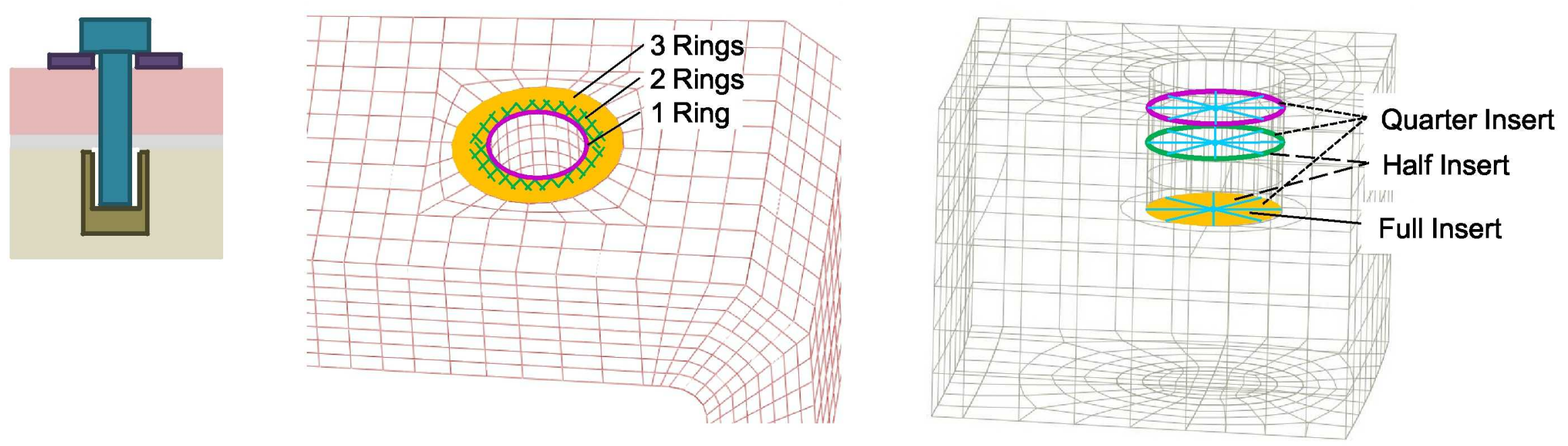

Initial

Modeling $\longrightarrow$

Assumptions

\begin{tabular}{|c|c|c|c|}
\hline Washer & Insert & Friction & Prediction \\
\hline 3 Rings & Full & 0.1 & 0.88 \\
\hline 2 Rings & Full & 0.1 & 0.98 \\
\hline 1 Ring & Full & 0.1 & 1.15 \\
\hline 3 Rings & Half & 0.1 & 0.91 \\
\hline 3 Rings & Quarter & 0.1 & 0.93 \\
\hline 3 Rings & Full & 0.04 & 0.88 \\
\hline 3 Rings & Full & 0.2 & 0.89 \\
\hline 2 Rings & Half & 0.1 & 1.02 \\
\hline
\end{tabular}




\section{Upper Stage LH2 Tank MHC Seal Joint Analyses}

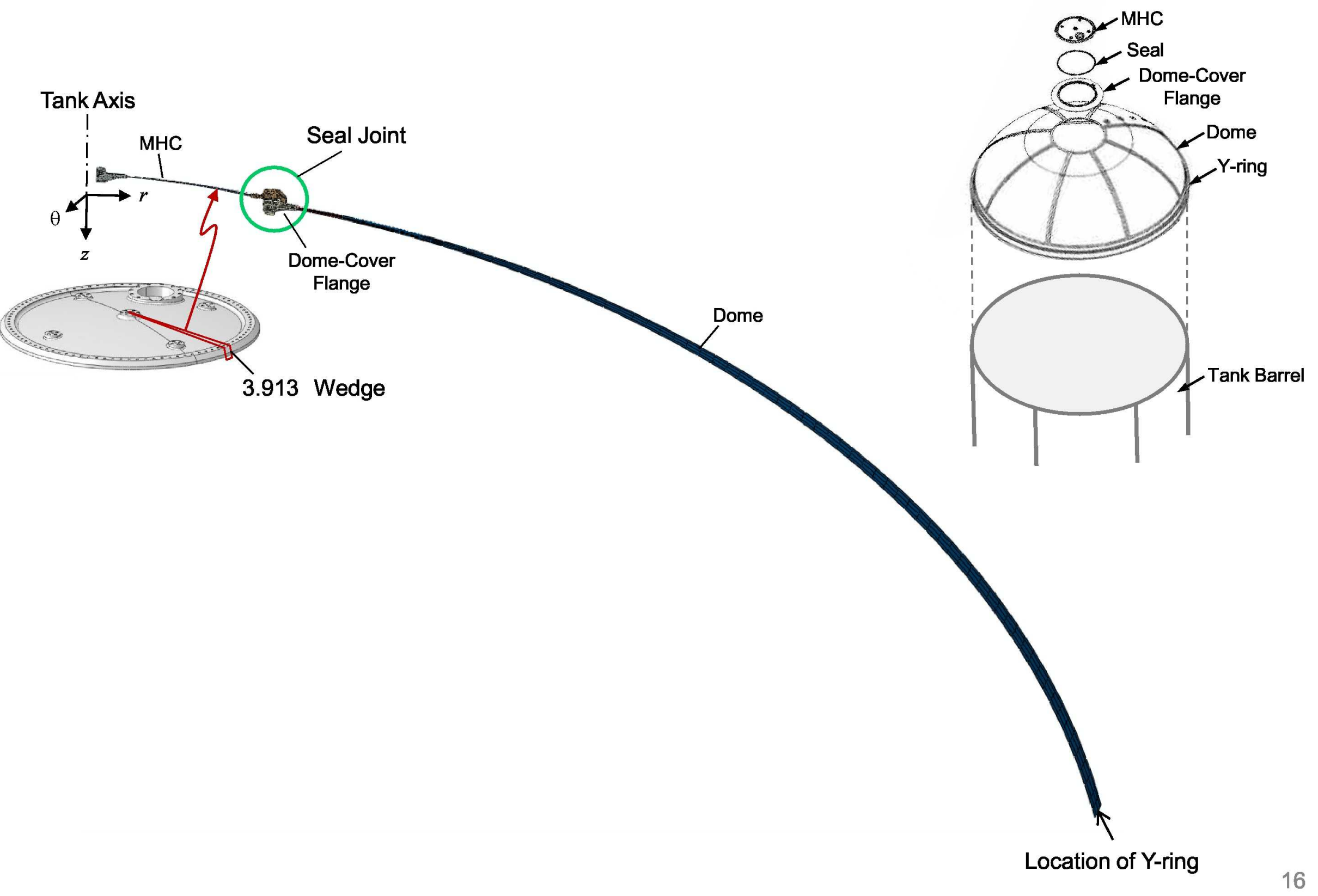




\section{Upper Stage Seal Joint Finite Element Model}

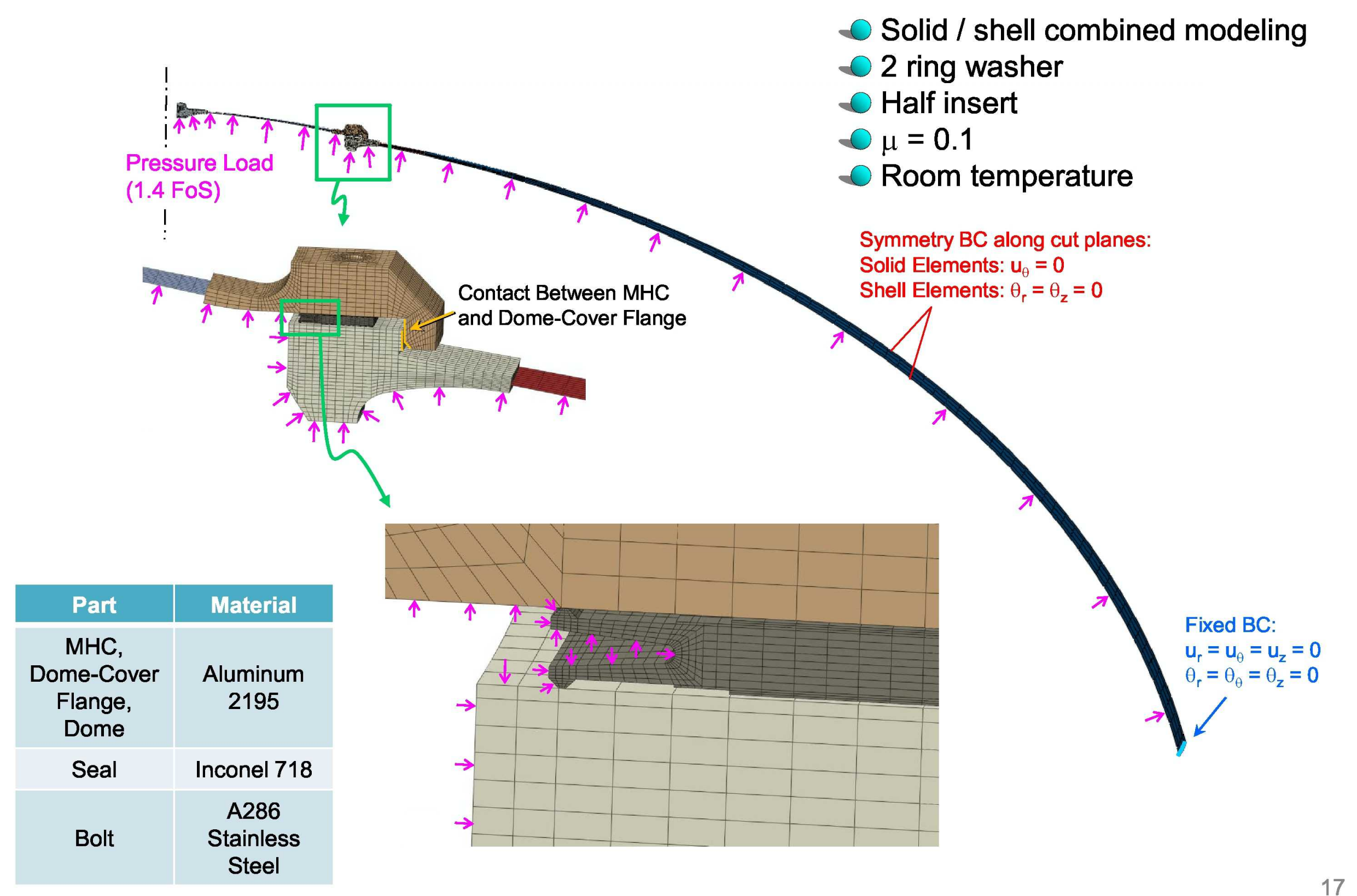




\section{Upper Stage Seal Joint Results}
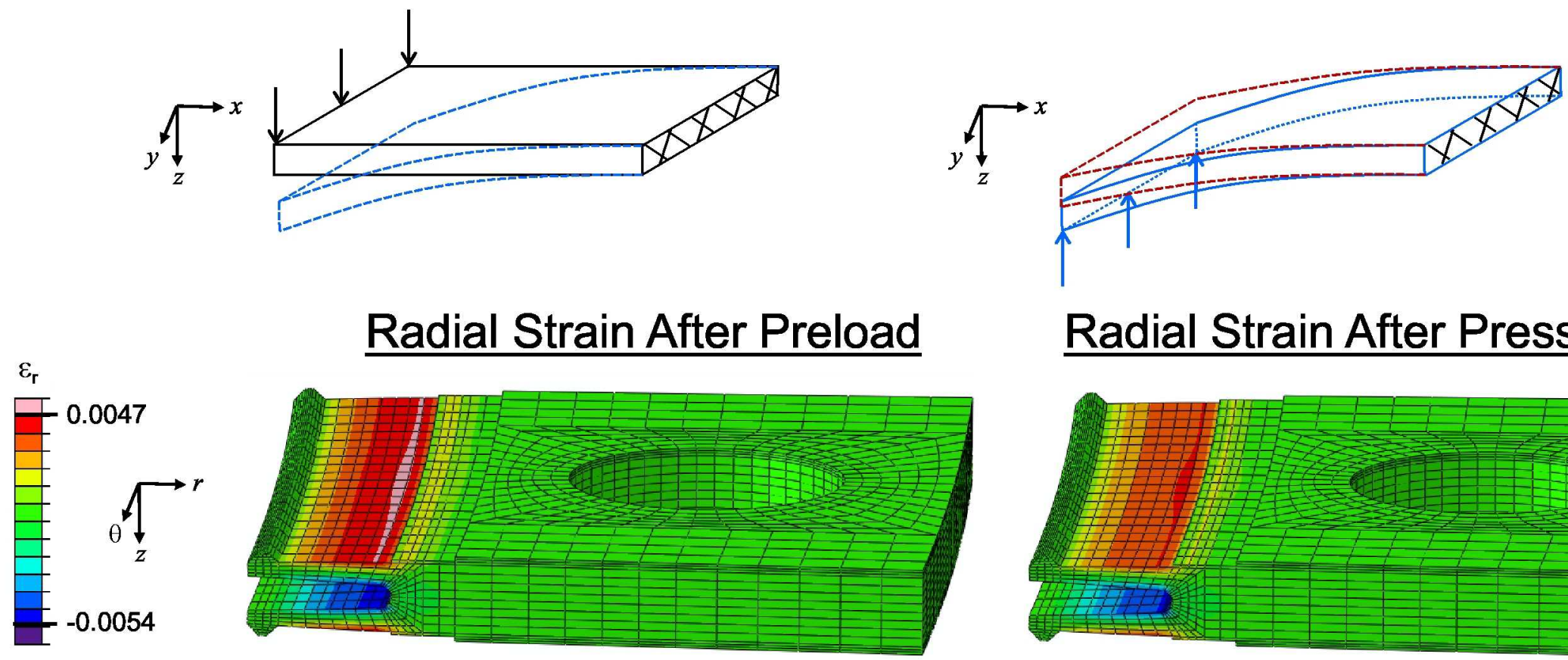

Radial Strain After Pressure Load

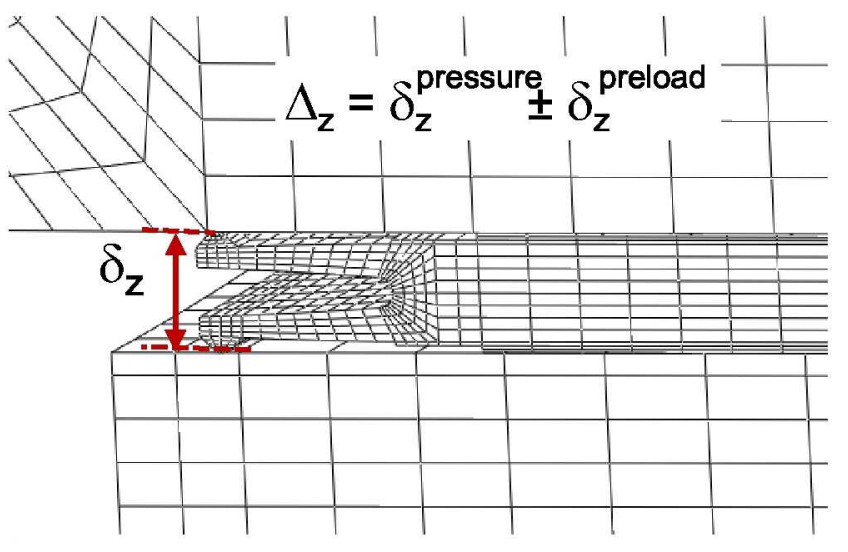

$\Delta_{z}$ / Allowable $=0.39$ (at Room Temp.)

- Preliminary results for cryogenic temperature indicate that joint opening is also within allowable. 


\section{Upper Stage Seal Joint Results}

Radial restraint $\left(u_{r}=0\right)$

$\underline{\text { Radial Restraint }}$

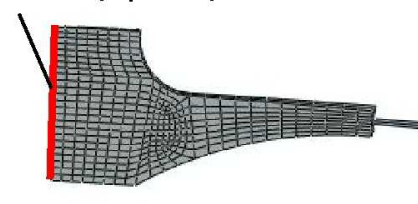

Negligible effect on joint opening

$\Delta_{z}$ / Allowable $=0.39$ (at Room Temp.)

Friction
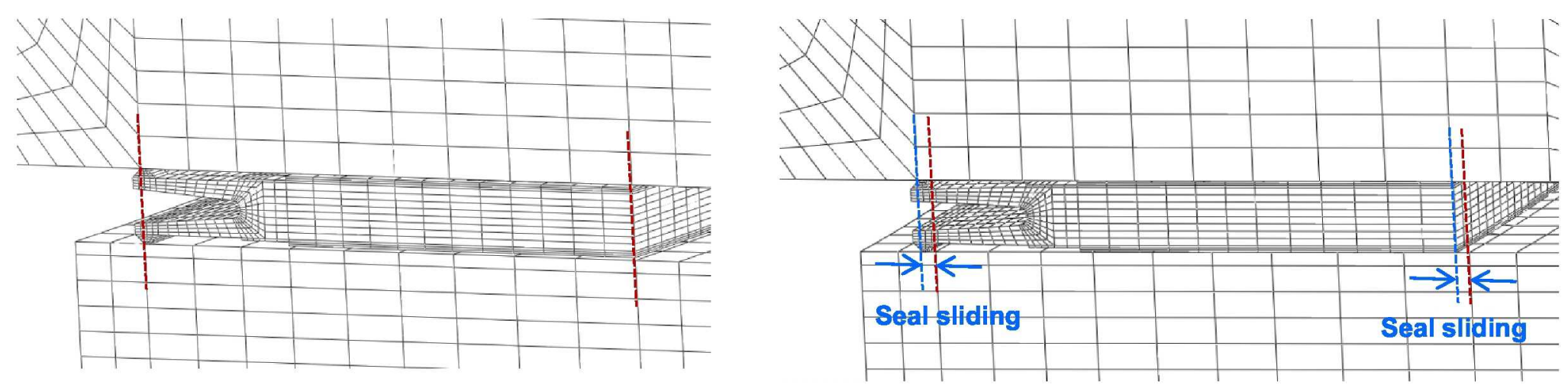

Negligible effect on joint opening

$\Delta_{z}$ / Allowable $=0.39$ (at Room Temp.) 


\section{Summary}

\section{Analysis Observations}

3D finite element modeling correlates well with test data

Demonstrated effects of modeling assumptions 


\section{Wedge Model of MHC}

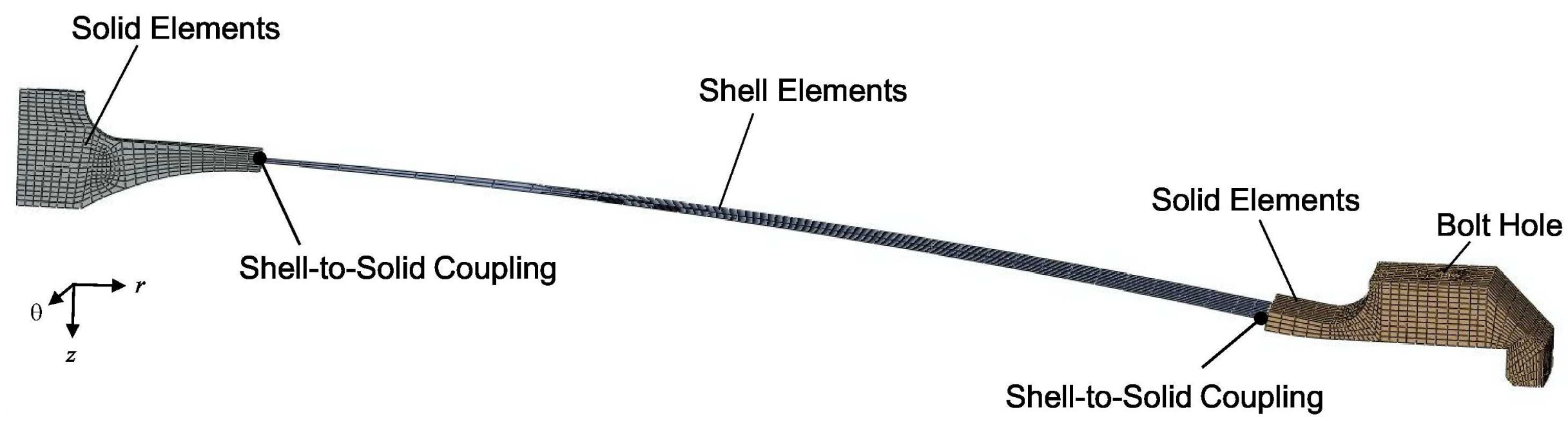




\section{Wedge Model of Dome-Cover Flange}

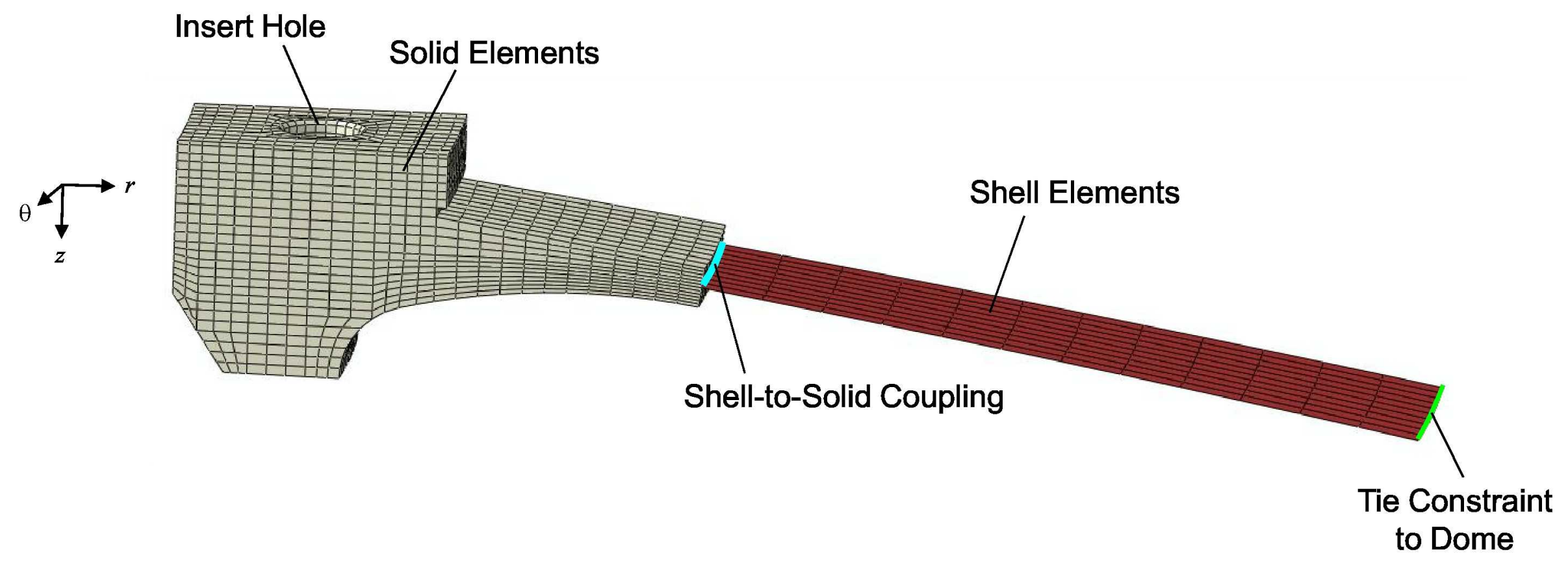

